$<$ Supporting Information $>$

\title{
Tunable Interlayer Distance via Adsorption of Cyclic Hydrocarbons in
}

\section{SCSC Mode}

Junhee Kim,${ }^{\dagger}$ Soojin Lee,${ }^{\dagger}$ Dongwon Kim,${ }^{\dagger}$ Dongwook Kim,, and Ok-Sang Jung $^{*}, \dagger$

'Department of Chemistry, Pusan National University, Busan 46241, Republic of Korea

$¥$ Center for Catalytic Hydrocarbon Functionalizations, Institute for Basic Science (IBS), Daejeon 34141, Republic of Korea

* Correspondence and requests for materials should be addressed to O.-S. Jung: oksjung@pusan.ac.kr 


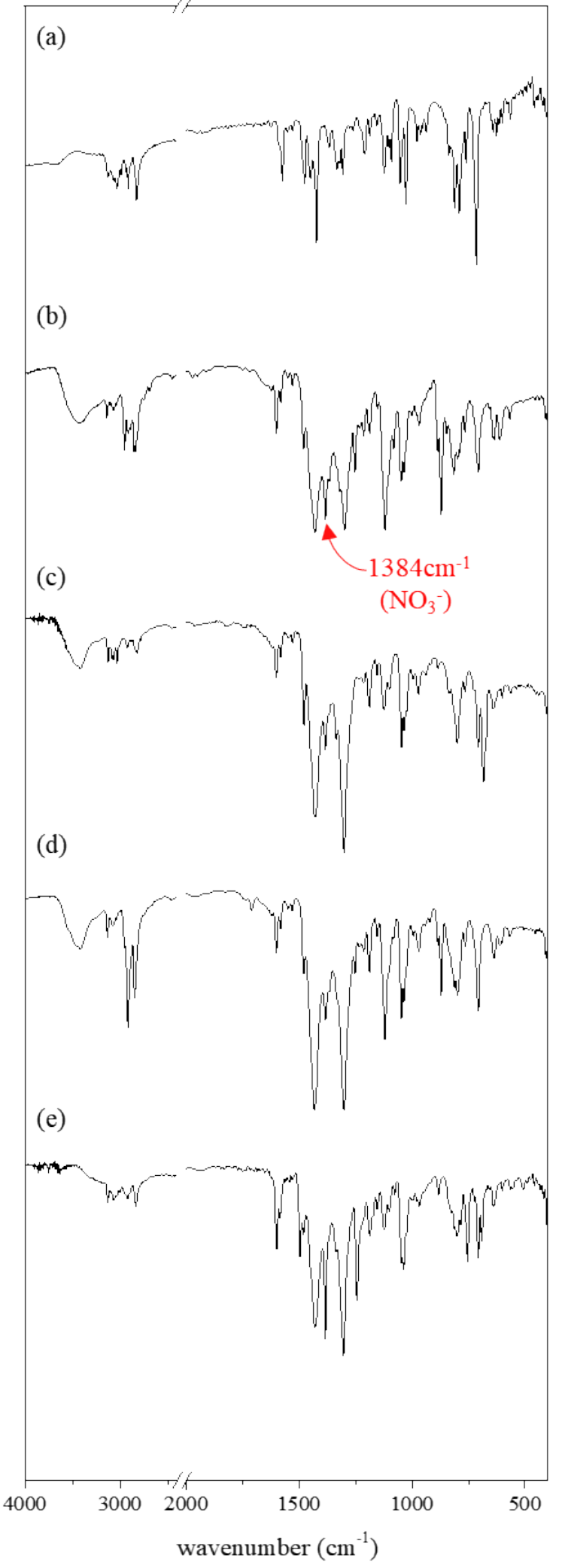

Figure S1. IR spectra of L (a), 4C $\mathrm{C}_{4} \mathrm{H}_{8} \mathrm{O}_{2} @\left[\mathrm{Cd}\left(\mathrm{NO}_{3}\right)_{2} \mathrm{~L}\right](\mathrm{b}), 4 \mathrm{C}_{6} \mathrm{H}_{6} @\left[\mathrm{Cd}\left(\mathrm{NO}_{3}\right)_{2} \mathrm{~L}\right]$ (c), $\mathrm{C}_{4} \mathrm{H}_{8} \mathrm{O}_{2} \cdot 0.8\left(\mathrm{C}_{10} \mathrm{H}_{18}\right) @\left[\mathrm{Cd}\left(\mathrm{NO}_{3}\right)_{2} \mathrm{~L}\right](\mathrm{d})$, and $4 \mathrm{C}_{7} \mathrm{H}_{8} \mathrm{O} @\left[\mathrm{Cd}\left(\mathrm{NO}_{3}\right)_{2} \mathrm{~L}\right](\mathrm{e})$. 

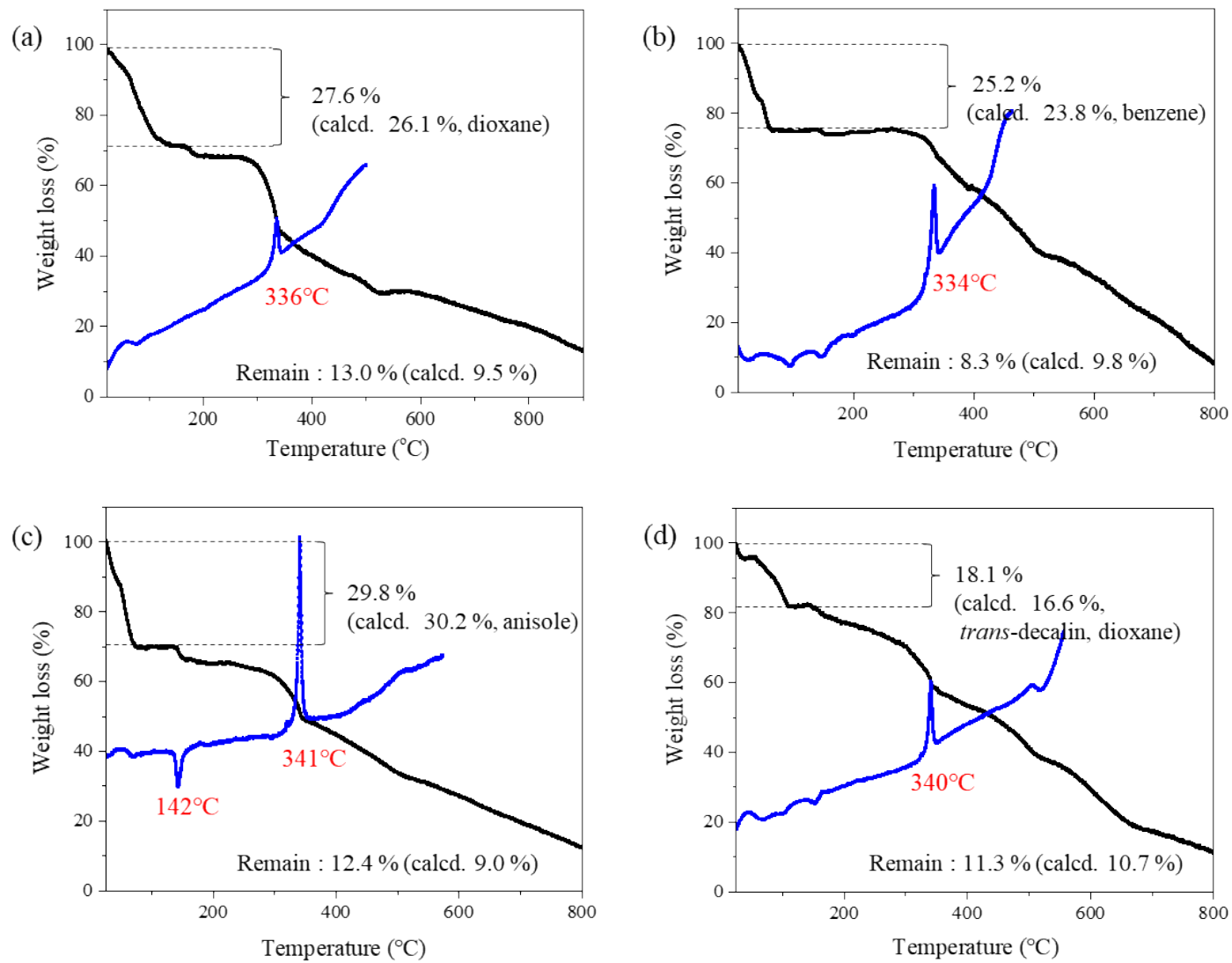

Figure S2. TGA and DSC overlay of $4 \mathrm{C}_{4} \mathrm{H}_{8} \mathrm{O}_{2} @\left[\mathrm{Cd}\left(\mathrm{NO}_{3}\right)_{2} \mathrm{~L}\right](\mathrm{a}), 4 \mathrm{C}_{6} \mathrm{H}_{6} @\left[\mathrm{Cd}\left(\mathrm{NO}_{3}\right)_{2} \mathrm{~L}\right]$ (b), $4 \mathrm{C}_{7} \mathrm{H}_{8} \mathrm{O} @\left[\mathrm{Cd}\left(\mathrm{NO}_{3}\right)_{2} \mathrm{~L}\right](\mathrm{c})$, and $\mathrm{C}_{4} \mathrm{H}_{8} \mathrm{O}_{2} \cdot 0.8\left(\mathrm{C}_{10} \mathrm{H}_{18}\right) @\left[\mathrm{Cd}\left(\mathrm{NO}_{3}\right)_{2} \mathrm{~L}\right](\mathrm{d})$. 

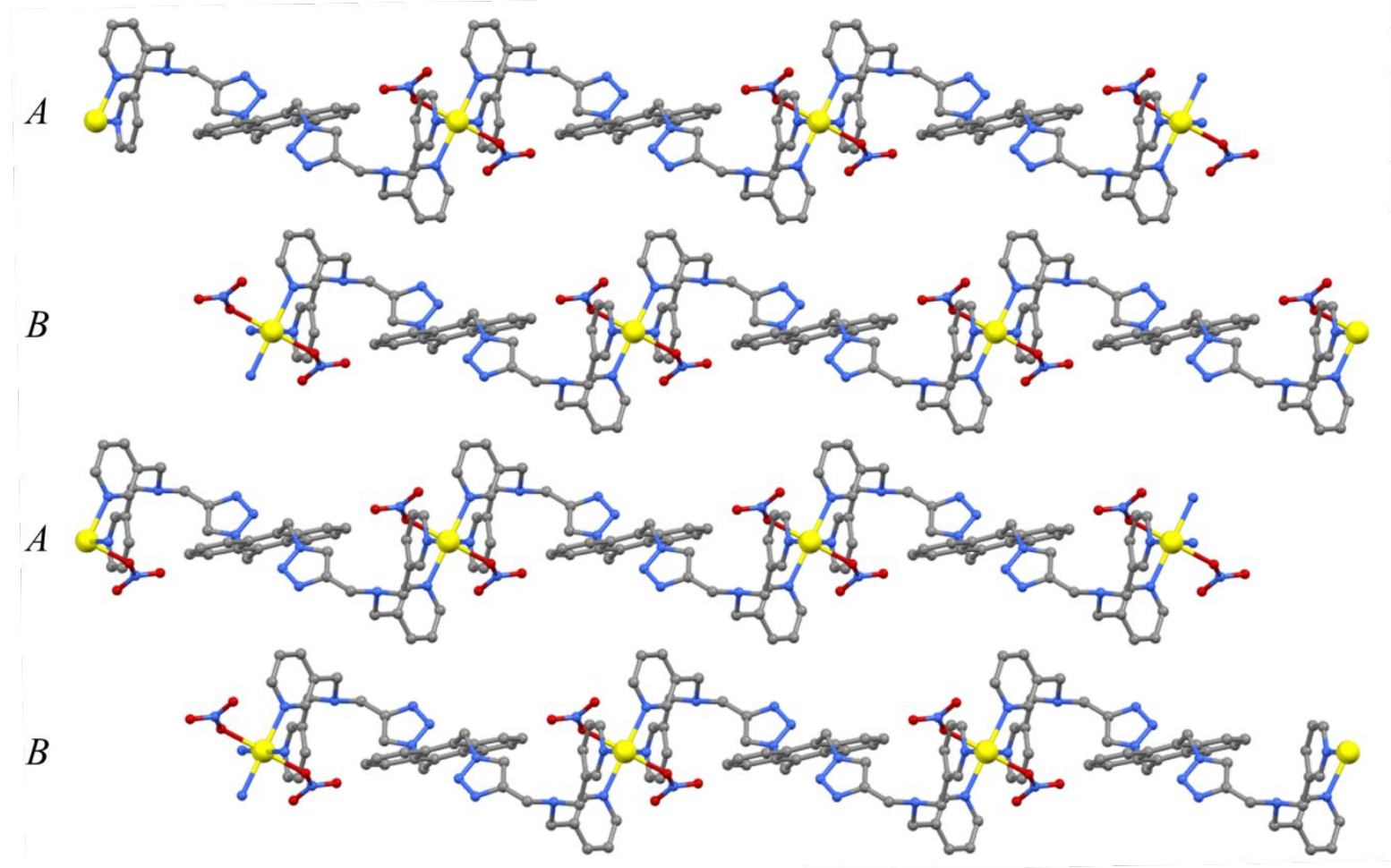

Figure S3. The crystal structure of $4 \mathrm{C}_{6} \mathrm{H}_{6} @\left[\mathrm{Cd}\left(\mathrm{NO}_{3}\right)_{2} \mathrm{~L}\right]$ showing an $A B A B$ mode. 
(a)

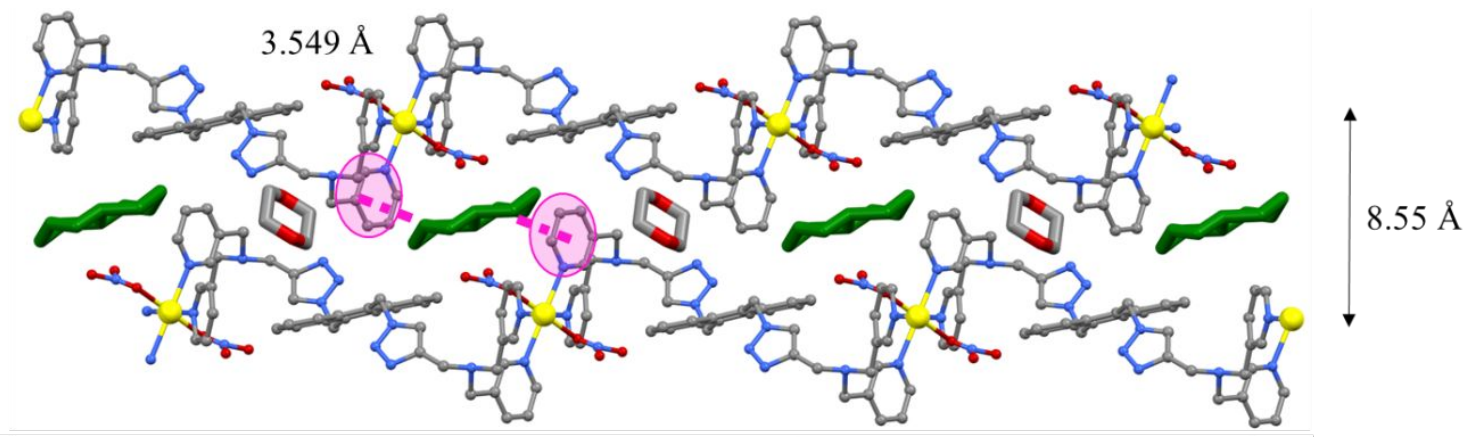

(b)

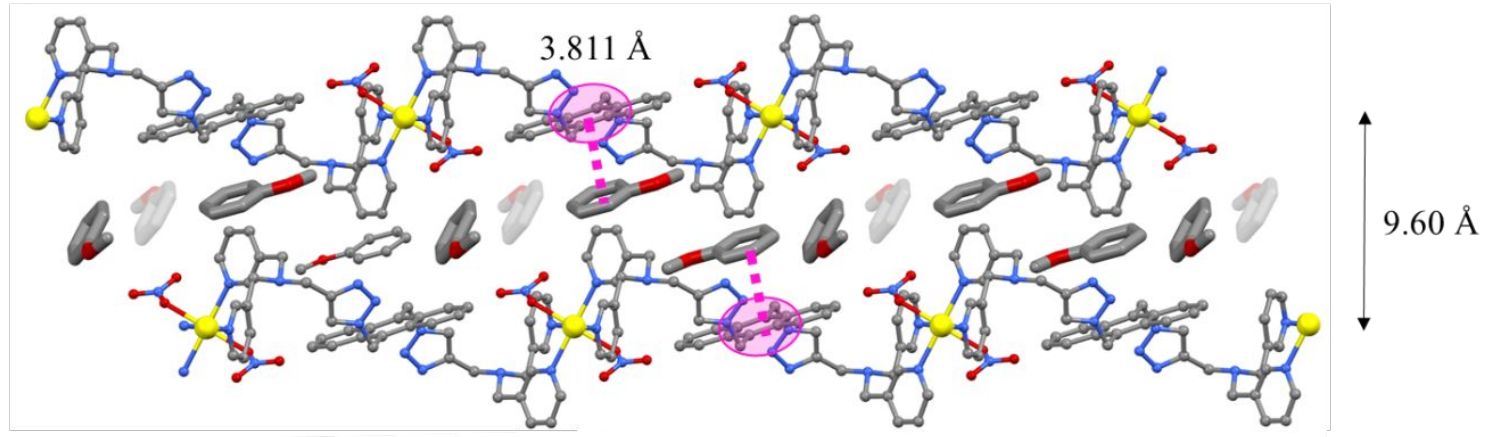

(c)

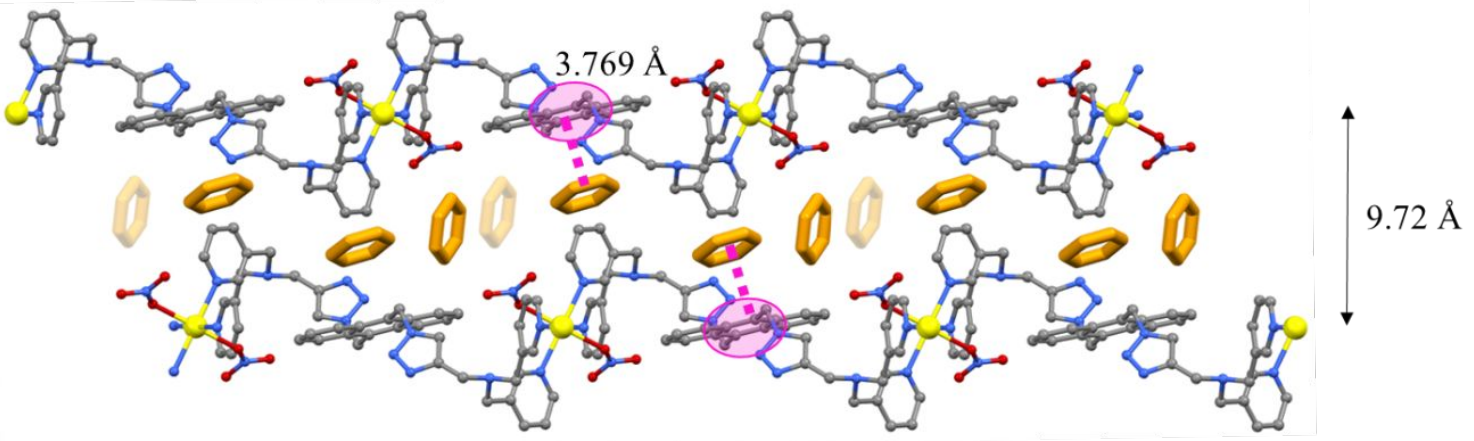

Figure S4. The crystal structures showing distance between $2 \mathrm{D}$ skeletons via guests; $\mathrm{CH}-\pi$ interaction (a), $\pi-\pi$ interaction (b), and $\pi-\pi$ interaction (c). 


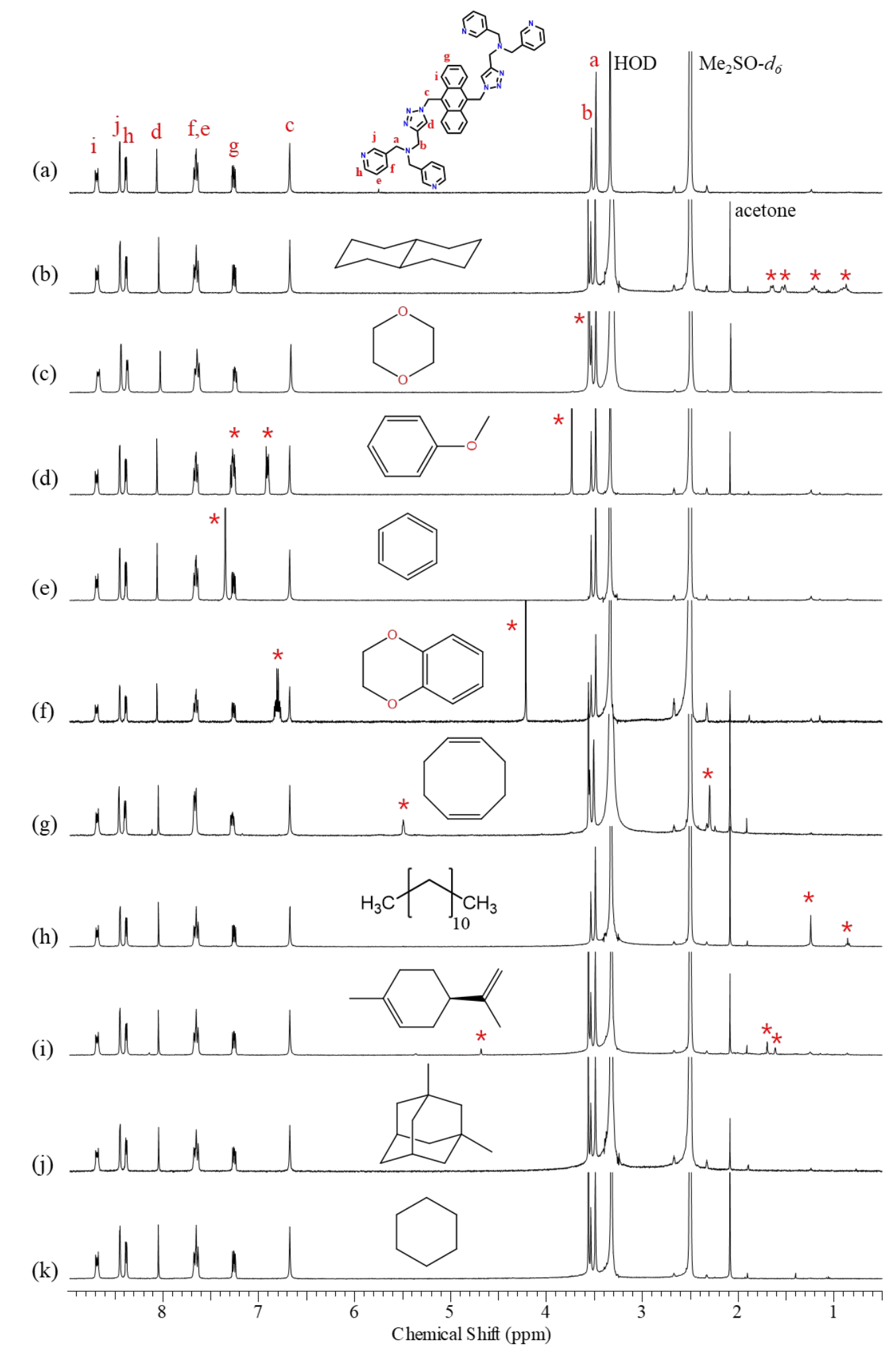

Figure S5. ${ }^{1} \mathrm{H}$ NMR spectra of (guest)@ $\left[\mathrm{Cd}\left(\mathrm{NO}_{3}\right)_{2} \mathrm{~L}\right]$ exchanged from $4 \mathrm{C}_{4} \mathrm{H}_{8} \mathrm{O}_{2} @\left[\mathrm{Cd}\left(\mathrm{NO}_{3}\right)_{2} \mathrm{~L}\right]$ (c). L (a), trans-decalin (b), anisole (d), benzene (e), 1,4benzodioxane (f), 1,5-cyclooctadiene ( $\mathrm{g}$ ), n-dodecane (h) and (R)-limonene (i), 1,3dimethyladamantane $(\mathrm{j})$, and cyclohexane $(\mathrm{k})$ as guests. For spectra $(\mathrm{j})$ and $(\mathrm{k})$, guest molecules were not adsorbed into the crystal. After the solvent exchange, the crystals were washed with acetone to wash off the solvents on the surface. 


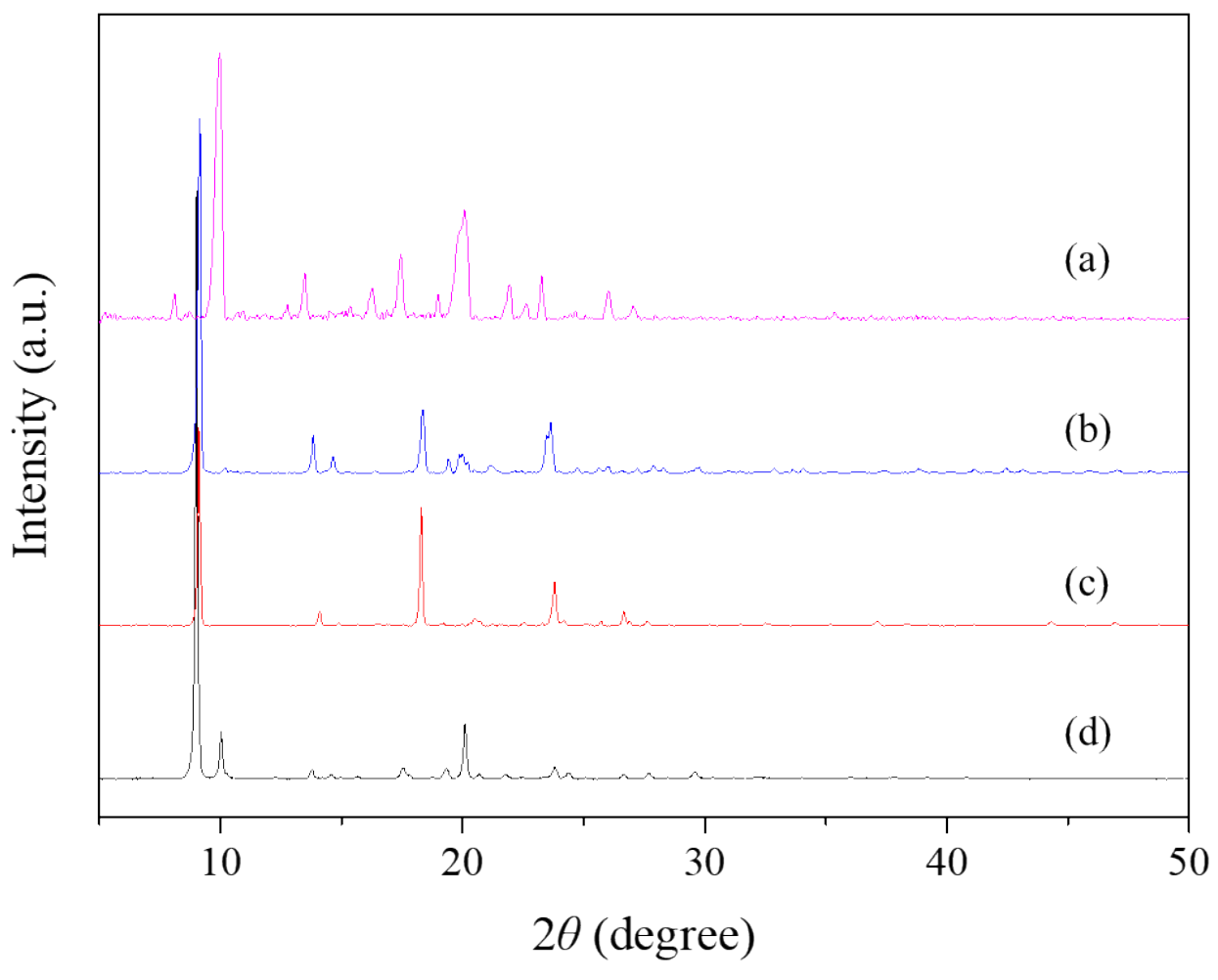

Figure S6. PXRD patterns of $\mathrm{C}_{4} \mathrm{H}_{8} \mathrm{O}_{2} \cdot 0.8\left(\mathrm{C}_{10} \mathrm{H}_{18}\right) @\left[\mathrm{Cd}\left(\mathrm{NO}_{3}\right)_{2} \mathrm{~L}\right]$ (a, $\mathrm{SCSC}$ exchanged), $4 \mathrm{C}_{4} \mathrm{H}_{8} \mathrm{O}_{2} @\left[\mathrm{Cd}\left(\mathrm{NO}_{3}\right)_{2} \mathrm{~L}\right]$ (b, as synthesized), $4 \mathrm{C}_{7} \mathrm{H}_{8} \mathrm{O} @\left[\mathrm{Cd}\left(\mathrm{NO}_{3}\right)_{2} \mathrm{~L}\right]$ (c, SCSC exchanged), and $4 \mathrm{C}_{6} \mathrm{H}_{6} @\left[\mathrm{Cd}\left(\mathrm{NO}_{3}\right)_{2} \mathrm{~L}\right]$ (d, SCSC exchanged). 